\title{
Femoral nonunion with segmental bone defect treated by distraction osteogenesis with monolateral external fixation
}

\author{
Qun Zhang*, Wei Zhang*, Zhuo Zhang, Licheng Zhang, Hua Chen, Ming Hao, Junhao Deng and Peifu Tang
}

\begin{abstract}
Background: Currently, the common treatment for femoral nonunion with large segmental bone defect is difficult and complex. The effective surgical methods are rare, include vascularized bone grafting, Masquelet technique and Ilizarov distraction osteogenesis. The objective of this study is to investigate the outcomes of segmental femoral defects treated with monolateral external fixation using the distraction osteogenesis.
\end{abstract}

Methods: We retrospectively analyzed patients with femoral nonunion with segmental bone defects $(>6 \mathrm{~cm})$ between January 2010 and January 2014 in our single trauma center. All patients were treated by distraction osteogenesis with monolateral external fixation. All surgeries were performed by the same surgeon. Bone union, duration of distraction osteogenesis in days, time to consolidation in months, external fixation index (EFI), complications, and additional surgical interventions were recorded postoperatively. The modified Application of Methods of Illizarov (ASAMI) criteria were used to evaluate the operative effectiveness.

Results: Forty-one patients were enrolled in this study for analysis. The length of the bone defect ranged from 6 to $17 \mathrm{~cm}$. All patients eventually achieved healing, and no patient experienced recurrence of infection or newly developed infection. The average time needed for healing was 13 months. In terms of the incidence of complications, 3 cases axial deviations, 5 cases docking site nonunion, 23 cases pin-tract infection, 14 cases knee joint stiffness or their joint mobility declined, 2 cases osteogenesis insufficient in the distraction area, 1 case refracture, and 2 cases loose external fixation pins. In terms of the evaluations of fracture healing and function, 30 patients excellent, 6 patients good, 5 patients fair, and 0 patient poor. In terms of postoperative function evaluations, 21 patients excellent, 9 patients good, 7 patients fair, and 4 patients poor.

Conclusion: For patients with femoral nonunion with large segmental bone defects, the monolateral external fixation can provide effective stability, improve compliance, and reduce complications.

Keywords: Monolateral external fixation, Distraction osteogenesis, Bone defects, Femoral nonunion

\section{Background}

The common causes of posttraumatic femoral nonunion with large segmental bone defect $(>6 \mathrm{~cm})$ include acute bone loss, bone ischemia atrophy in nonunion sites, and surgical removal of dead bone and sclerotic bone after infection [1, 2].Current treatment for the disease, in addition to the need of addressing the issue of bone nonunion with bone defect, soft tissue defect, nearby

\footnotetext{
*Correspondence: zhang_qun2017@163.com; zwtyrran@163.com

Qun Zhang and Wei Zhang are co-first authors.

Qun Zhang and Wei Zhang contributed equally.

Department of Orthopaedics, Chinese PLA General Hospital, No. 28 Fuxin

Road, Beijing 100853, People's Republic of China
}

joint stiffness, deformities (rotation, angulation, and shortening), infection and many other issues should also be treated simultaneously [3-5]. At present, the common treatments include vascularized bone grafts (such as ribs, ilium, and fibula), intramembranous osteogenesis technique (Masquelet technique), and Ilizarov distraction osteogenesis [6-10]. Among them, Ilizarov distraction osteogenesis can simultaneously address the issues of infection, bone and soft tissue defects, and correction of deformities and eventually achieves the fracture healing. It is one of the most effective therapeutic strategies for posttraumatic complex nonunion $[5,9,10]$.Clinically, 
the Ilizarov circular frame, the Taylor spatial frame (TSF), the semicircular Ilizarov pin fixator, and the conventional external fixation are applied to distraction osteogenesis [11-15]. However, complications associated with external fixation systems are high. Moreover, the compliance of patients is relatively poor. Although the monolateral external fixation can provide good stability and compliance is high, there are relatively few reports that describe the outcomes of femoral nonunion treated with this external fixation system [16-18].

Therefore, we retrospectively analyzed patients with femoral nonunion with segmental bone defects who were treated with the monolateral external fixator between January 2010 and January 2014 to evaluate the effectiveness, stability, and complications of the monolateral external fixator.

\section{Methods}

The inclusion criteria were as follows: (1) posttraumatic femoral nonunion, (2) segmental bone defect $>6 \mathrm{~cm}$ preoperatively and/or intraoperatively, and (3) Ilizarov technique with the monolateral external fixator. Patients who met the above criteria were included in this study. The exclusion criteria were as follows: (1) nonunion caused by primary or secondary tumor, congenital bone disease, metabolic bone disease, or severe vascular origin disease; (2) nonunion for which physiotherapy or drug therapy was used during the treatment to promote fracture healing; (3) nonunion that was combined with severe systemic organ failure; and (4) nonunion that was combined with a mental disorder.

\section{Surgical procedure}

After successful anesthesia, for patients who originally had fixation objects, the internal and external fixation objects were firstly removed to expose the nonunion site (Fig. 1a-g2). Then, dead bone, sclerotic bone, fibrous scar tissue, and infected tissue were completely debrided, and a pendulum saw was used to clean up the nonunion site until fresh bleeding healthy bone tissue was reached. By now, the length of bone defect was measured. On the basis of radical debridement, the placement of external fixation pins was initiated. The hydroxyapatite-coated external fixation pins and the extendable monolateral external fixation frame were from Orthofix, Italy. Under the image intensifier, 8-9 parallel pins were inserted at the lateral side of the femur, perpendicular to its long axis. Three pins were fixed at the proximal and distal ends of the femur, respectively, and 2-3 pins were inserted on the transported bone segment. Make sure all pins are on the same coronal plane. The external extendable monolateral fixation frame was then installed. After the osteotomy plane was determined, smallincision low-energy subperiosteal osteotomy was

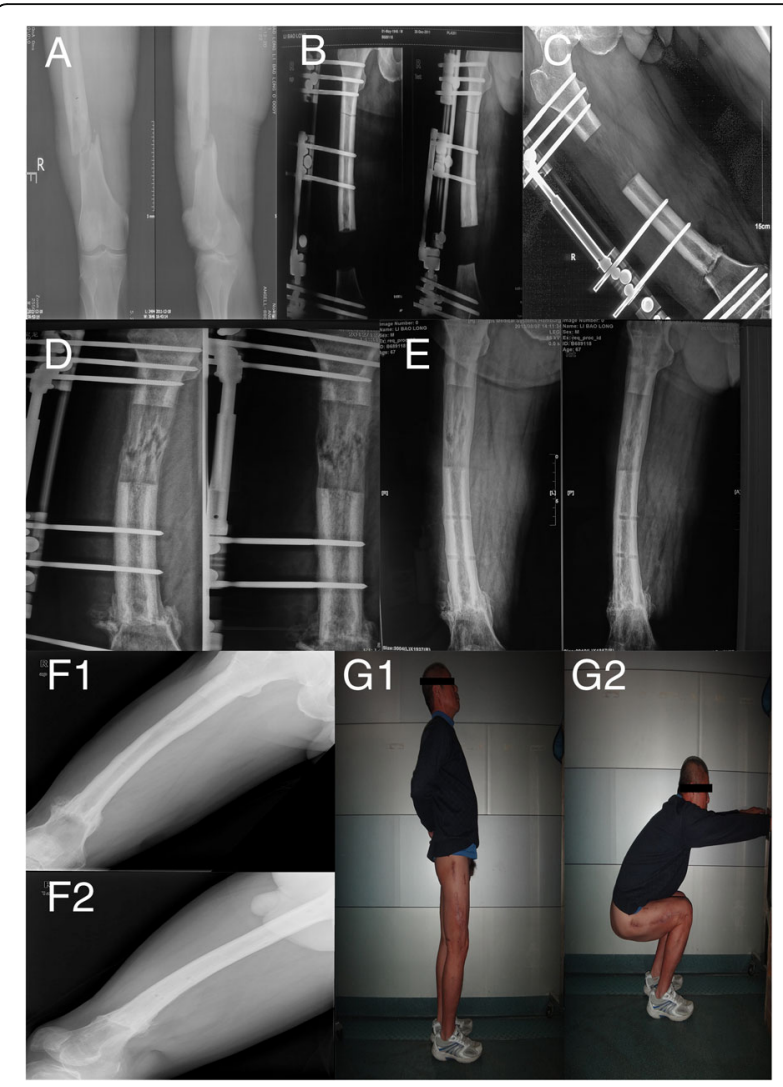

Fig. 1 Sixty-year-old male, suffered a 2-year-long postoperative infection after fracture of left femoral shaft. a The X-ray showed refractures occurred 2 years later; $\mathbf{b}$ the patient was given the debridement of lesions, single-arm external fixator, and bone transport. The postoperative presentation of the $\mathrm{X}$-ray demonstrated a 10-cm-long bone defect of femur. c The 5-month-later presentation of the $\mathrm{X}$-ray demonstrated the femur length became normal. $\mathbf{d}$ The 1 -year-later presentation of the X-ray demonstrated the bone grew well in the region of distraction osteogenesis and the docking site healed well. e The 18-month-later presentation of the X-ray with external fixator removed. $\mathbf{f}$ The 4-year-later $X$-ray presentation showed no infection recurred. $\mathbf{g}$ The patient showed a good function of flexion and extension of knee joint

performed, and compression was applied at the osteotomy site. Finally, the incision was closed. If the soft tissue could not be closed during the primary phase or infection was severe, a vacuum-assisted closure device was used to cover the wound, and the wound would be closed during the second phase. The suspected tissue was taken from multiple sites during the procedure and was sent for bacteriological culture to guide postoperative antibiotic use. The surgeries were completed by the same experienced senior surgeon.

\section{Postoperative management}

For aseptic nonunion, patients were treated with broadspectrum antibiotics for 3 days. For septic nonunion, anti-infection treatment was administered for 14 days 


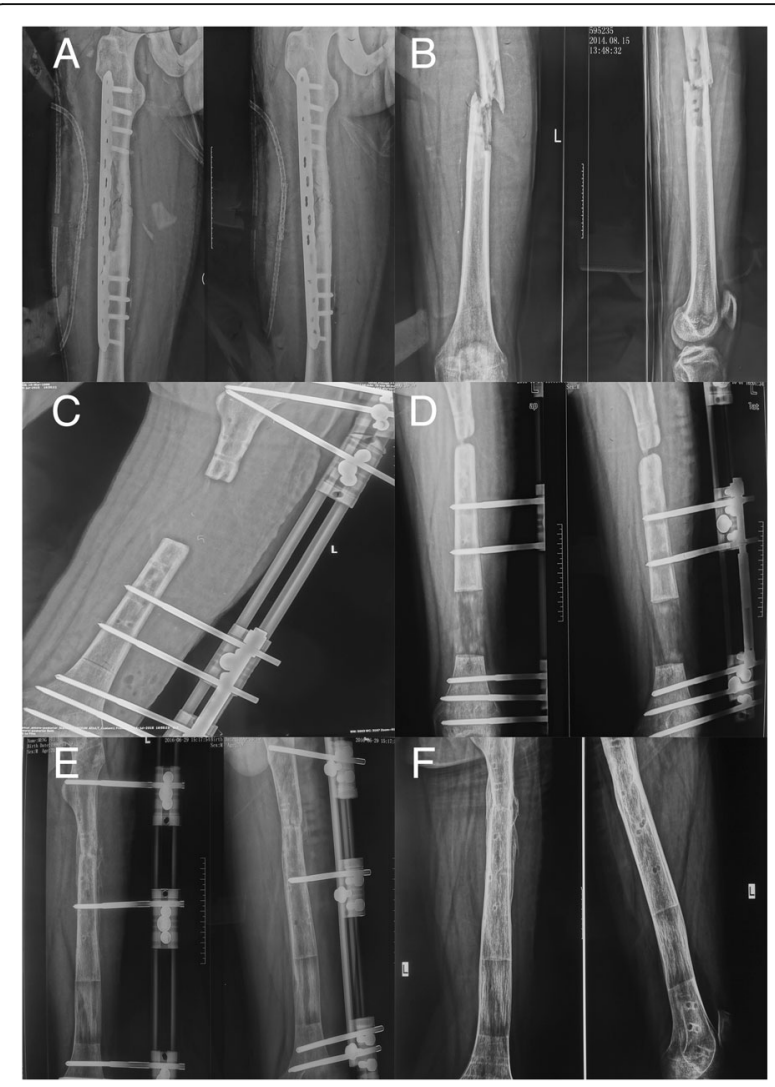

Fig. 2 Twenty six-year-old male, suffered a 14-month-long infection after the operation using bone plates of fracture of left femoral shaft. a The X-ray presentation after the open debridement combined with irrigation outside the hospital. b After removing the internal fixator due to the runaway infection, the postoperative presentation of the X-ray demonstrated the nonunion of fracture and evident displacement, and some sequestra with bone defects could be seen locally. c The 1-week-later X-ray presentation after bone transport: single-arm external fixator served well, and there existed a 6-cm-long bone defect after thorough removal of sequestra and infectious tissues. d The 2-month-later X-ray presentation after bone transport: bone growth could be seen in the region of distraction osteogenesis, but both sides of docking site were significantly hardened. e The 14-month-later X-ray presentation after bone transport: the docking site healed well after debridement, autogenous bone graft, and compression. The bone grew well in the region of distraction osteogenesis and external fixation pins were partly removed. $\mathbf{f}$ : The 20 -month-later $\mathrm{X}$-ray presentation after bone transport: no infection recurred

according to the drug sensitivity results (Fig. 2a-f). Disinfection care for the pin tract was conducted every day to prevent infection. Seven to 10 days postoperatively, bone transport was initiated, with the extension based on $0.25 \mathrm{~mm}$ four times per day. Three days postoperatively, the patients began partial weight-bearing activities with crutches. X-rays were taken and reviewed every 2 weeks to observe the growth condition of the distraction area and whether there was axial deviation of the transported bone segment. X-ray monitoring was stopped when the limb length was achieved or when the docking site made contact. Before removing the external fixator, the compression or distraction force was gradually eliminated to ensure that the frame connection was neutral so that there was no tension in any direction. The removal of the external fixation was based on the following findings: osteogenesis is sufficient in the distraction area, fracture healing was reliable, and no deformations were found at the nonunion site and distraction area when the patient walked on full weight-bearing activities.

All relevant complications were recorded, and the corresponding treatments were clarified. Postoperative pintract infection was classified according to Marsh's description [19]. The assessment of clinical efficacy was conducted by the modified Application of Methods of Illizarov (ASAMI) criteria [20].

\section{Results}

According to the inclusion and exclusion criteria, our study enrolled 43 patients (Additional file 1). Two patients were lost to follow-up, and 41 patients were eventually included for analysis. The patients ranged in age from 26 to 76 years old, with an average age of 44 years old. There were 31 males and 10 females. Twenty-eight patients had previous open fractures, and 13 patients had previous closed fractures. The duration from the time of injury to the present ranged from 10 to 60 months, with an average of 23.4 months. The patients had received 1-9 previous surgeries: 7 patients had initial internal fixations, 21 patients had intramedullary (IM) nailing, and 13 patients had external fixation. The length of the bone defect ranged from 6 to $17 \mathrm{~cm}$, with an average length of $10.1 \mathrm{~cm}$. There were 33 cases of septic nonunion (21 draining and 12 quiescent nonunion) and 8 cases of aseptic nonunion. Fifteen injured limbs had combinations of rotation deformities, and 11 cases had angulation deformities. Eight patients had knee joint dysfunction. The demographic characteristics of the patients studied can be seen in Table 1.

The postoperative follow-up time ranged from 20 to 60 months (average 35 months). All fractures eventually achieved healing, and no patient experienced recurrence of infection or newly developed infection. The duration of distraction osteogenesis (DOG) was 60-191 days (average 110 days). The time needed for healing was 620 months (average 13 months). The external fixation index (EFI) was 1.15-1.52 months/cm (average 1.30 months/cm). In terms of complications, 3 patients had axial deviations, which were corrected by surgical adjustment and enhanced fixation; 5 patients had docking site nonunion, of whom 3 patients were given autologous cancellous bone grafting combined with continuous compression at the docking site to achieve healing, and 2 patients underwent the "accordion technique" to achieve healing. There were 23 cases of pin- 
Table 1 Demographic characteristics of the patients studied

\begin{tabular}{ll}
\hline Variable & Number \\
\hline Total number & 41 \\
Age (years) & $26-76$ \\
Gender & 31 \\
Male & 10 \\
Female & $10-60$ \\
Time since injury (months) & $1-9$ \\
Number of surgeries & \\
Patterns of initial fractures & 28 \\
Open & 13 \\
Closed & \\
Patterns of initial surgeries & 7 \\
Plating & 21 \\
IM nail & 13 \\
External fixation & \\
Patterns of bone nonunion & \\
Infection & 33 \\
Aseptic & 8 \\
Length of bone loss (cm) & 11 \\
Other types of deformities & \\
Rotation & Angular \\
\hline
\end{tabular}

tract infection, of which 15 cases were type A, 7 cases were type $\mathrm{B}$, and 1 case was type C; 10 of those cases mainly occurred at the greater trochanter site, while the pins of 3 patients were removed and replaced, after which control was gained over the infection. Fourteen patients had knee joint stiffness and a range of knee motion declined; according to the differences in the patients' living requirement, joint arthrolysis was performed later for 10 patients. Two patients had osteogenesis insufficient in the distraction area; 1 patient was treated with autologous cancellous bone grafting, while the other patient was treated with the "accordion technique." One patient experienced refracture when he accidentally fell down 1 year after the external fixation frame was removed; the refracture occurred at the docking site. Since this patient previously had an infectious bone nonunion, an external fixation frame was used for fixation again, and refracture healing was achieved with the "accordion technique." Two patients had loose external fixation pins, which were replaced with new fixation pins (see Table 2). In terms of the evaluations of fracture healing and function, 30 patients excellent, 6 patients good, and 5 patients fair. In terms of postoperative function evaluations, 21 patients excellent, 9 patients good, 7 patients fair, and 4 patients poor (see Table 3 ).
Table 2 Details of the outcomes and complications

\begin{tabular}{ll}
\hline Variable & $35(20-60)$ \\
\hline Follow-up in months & $110(60-191)$ \\
Duration of DOG in days & $13(6-20)$ \\
Time to consolidation in months & $1.30(1.15-1.52)$ \\
EFI (months/cm) & 41 \\
The number of union & \\
The number of complications & 23 \\
Pin-track infection & 2 \\
Wire/pin loosening & 0 \\
Reinfection in fraction site & 0 \\
Vascular/nerve injury & 3 \\
Axial deviation & 5 \\
Docking site nonunion & 1 \\
Refraction & 2 \\
Osteogenesis insufficient in distraction area & 14 \\
Knee joint rigidity & 3 \\
The number of additional surgical interventions & \\
Bone grafting & 4 \\
Knee arthrolysis & 10 \\
Accordion technique & 3 \\
External fixator adjustment & \\
Remove/change external fixation pin & \\
Fixation in refraction & \\
\hline DOG distacton osteggnesis, Ef extenat fixaton index & \\
\hline
\end{tabular}

DOG distraction osteogenesis, EFI external fixation index

\section{Discussion}

The incidence of femoral nonunion is increasing, with a recent report published in JAMA indicating that it is as high as $13.9 \%$ [21]. This increase may be related to the increasing number of patients with severe fractures (higher degrees of open and comminuted fracture) caused by high-energy injuries (traffic, high-level fall, and crush injuries). When this condition is combined with large bone segment defects $(>6 \mathrm{~cm})$ and infection, it becomes even more difficult to solve [1]. Traditional treatment requires multiple operations at different

Table 3 Evaluation of the bone and functional results

\begin{tabular}{lll}
\hline Grades & Bone results $^{\mathrm{a}}$ & Functional results $^{\mathrm{b}}$ \\
\hline Excellent & 30 & 21 \\
Good & 6 & 9 \\
Fair & 5 & 7 \\
Poor & 0 & 4 \\
\hline
\end{tabular}

${ }^{a}$ Excellent result was defined as union, no infection, deformity of $7^{\circ}$ and limb length discrepancy (LLD) of $2.5 \mathrm{~cm}$; good was defined as union, with any two of the other three criteria; fair result was defined as union, with one of the other three criteria; and poor result was defined as nonunion

${ }^{b}$ Excellent result was defined as active, without the other four criteria; good was defined as active, with 1-2 of the other four criteria; fair was defined as active, with 3-4 of the other four criteria; and poor was defined as inactive 
stages. Only under the premise that a thorough debridement is conducted to control the infection or there is clearly no infection can the next step in the treatment strategy for bone defect repair be determined. Traditional surgical methods often cannot effectively and simultaneously solve a series of problems including bone and soft tissue defects, lower limb deformity (rotation, angulation, and shortening), fracture nonunion, and infection.

In terms of femoral nonunion with segmental bone defects, the frequently used treatment methods include vascular pedicle autologous bone grafting (such as ribs, ilium, and fibula), intramembranous osteogenesis technique (Masquelet technique), and Ilizarov distraction osteogenesis. All of these methods have their advantages and limitations. Autologous bone grafting with a vascular pedicle requires high level of microsurgical techniques; the bone supply is limited, and it will cause a secondary damage to the donor site; failure of revascularization of the transplanted bone segment will lead to the failure of fracture healing, and insufficient femoralization of the transplanted bone segment will result in poor bone strength, which then becomes prone to refracture $[6,7,22]$.

Compared with the above traditional surgical method, the Masquelet and Ilizarov techniques are the main surgical methods for the treatment of large segmental bone defects of the femur [9]. With the internal fixation, the Masquelet technique allows the patients to avoid carrying a bulky circular external fixator and its associated complications, thereby increasing patient's compliance. However, this technique has a higher requirement for the integrity of muscle soft tissue; it requires multiple operations (at least 2) and a large amount of autologous bone; it has a higher risk of reinfection and failure of revascularization and ossification of the transplanted bone region; and it is poor at correcting severe deformities $[8,9,23]$. Therefore, its surgical indications should be selected cautiously and strictly. The Ilizarov technology has unique advantages in the treatment of femoral nonunion, especially with large-segment bone defect, as it can simultaneously address infection, bone and soft tissue defects, and corrections of deformities at the primary stage. It is suitable for various types of nonunion with a lower requirement for soft tissue covering and a higher fracture healing rate [9-12, 16-18]. Our study achieved a $100 \%$ fracture healing rate, and the functional rates of good/ excellent were achieved by $73.2 \%$ of patients.

The common external fixation systems with the Ilizarov technique include the Ilizarov circular frame, the TSF, the semicircular Ilizarov pin fixator, and the conventional external fixator. However, they are often full-circular or hybrid external fixation frames, which are bulky for patients to carry and affect the exercise of adjacent joints; they also have high demands in terms of the surgeon's technique. Therefore, Harshwal used monolateral external fixation frames to treat 7 cases of femoral nonunion, and 5 cases achieved fracture healing with good function [16]. R. Rohilla compared monolateral external fixation with circular external fixation for the treatment of tibial nonunion and found that although the circular external frame could provide better stability with less screw path infection, there were no significant statistical differences in terms of fracture healing rate and functions in the two groups [24].

On the basis of our own study, we found that the monolateral external fixator has the following advantages: (1) the surgical procedure is simpler, and it is easy to promote to lower-level medical trauma centers; (2) patients have a better tolerance, and the functional exercises are more convenient, which can facilitate improved knee joint function so that the patient can return to family and society earlier; (3) it is more suitable for patients with senile or disuse osteoporosis, and the hydroxyapatite-coated external fixation pins have stronger holding power so that the risks of loosening and failure of cutting are probably lower; and (4) since the external fixation pins are fixed from the lateral side of the femur, the risk of neurovascular damage may be lower than that associated with the circular external frame. The drawback is that, compared with the circular external frame, when the soft tissue coverage of the healthy active bone tissue at the proximal and distal femur is insufficient or when the affected limb has deformities in all three-dimensional planes, the monolateral external fixation frame cannot be used. All of the 41 patients in our study group used the monolateral external fixation frame and could perform out-of-bed functional exercises early to achieve fracture healing; furthermore, the incidence of complications was lower.

It has been reported that when distraction osteogenesis was used for the treatment of femoral nonunion, the incidence of surgical complications was very high $[12,18,25,26]$, with the mean complications per patient ranging from $1.33(20 / 15)$ to 3.55 $(71 / 20)$ [18]. Our study result was $1.22(50 / 41)$, and the average length $(10.1 \mathrm{~cm})$ of bone defects of the included patients was much larger than the lengths in previously reported studies $(6-8.3 \mathrm{~cm})$. Under a much longer treatment cycle and with more complex conditions, the incidence of complications of this study was even lower. The most common complication was still pin-tract infection, though the incidence (56.1\%) of pin-tract infection in our study was also lower than those described in other reports (63-100\%) [25]. This difference may be associated with better patient education, more stable fixation by external fixation 
pins, and less interference with soft tissue (the use of the monolateral external fixation avoids contralateral soft tissue piercing). The second common complication was knee joint stiffness. In our study, 8 patients had preoperative joint stiffness, and only 6 patients had newly developed postoperative joint stiffness or functional decline. This low incidence was probably because there was no obstruction by the external frame behind the knee joint with the monolateral external fixation, which allows the patients to be able to perform early postoperative full-range joint exercise. However, for patients with preoperative joint stiffness, circular or hybrid external fixation also have unique advantages. The surgeon can simultaneously place a trans-articular external fixation to perform traction treatment on the stiff joint, which is a defect of the monolateral external fixation. The surgeon can choose a circular external fixation to perform simultaneous correction of joint stiffness at the primary stage according to the condition of the patient's joint function and the surgical requirement, or the surgeon can perform joint release or joint traction at the second stage after fracture healing.

In addition, the incidence of other complications is relatively lower. However, we should pay more attention to docking site nonunion and insufficient osteogenesis in the distraction area, especially in longer bone defect and poorer soft tissue condition and elderly patients with poorer osteogenic capacity. The "accordion technique" is an effective method. By giving repeated compression-distraction stimulation at the docking site or distraction area, it can induce intramembranous and endochondral osteogenesis, thereby promoting fracture healing [27]. When necessary, autologous iliac bone graft can also be a surgical option, but this procedure is often associated with damage to the donor area and insufficient bone supply. When the lengthening bone segment experiences axial deviation, an external fixation should be adjusted immediately. By increasing the contact area of the docking site and correcting poor alignment of the affected limb, the incidence of docking site nonunion can be reduced. If necessary, a small incision can be made to clean up any fibrous scar tissue at the docking site, and a limited decortication can be performed to improve the rate of fracture healing $[5,28]$.

This study also has some limitations. First, this study is a retrospective small-sample single-center study with a low level of evidence. Secondly, since there is no control group, we can only evaluate the advantages and shortcomings of the monolateral external fixation, which cannot prove that it is superior to circular external fixation. All of these observations require further confirmation in large-sample multi-center prospective randomized controlled trials.

\section{Conclusion}

Compared with the traditional circular or hybrid external fixation, even for patients with large segmental femoral defects, the monolateral external fixation can provide effective mechanical stability, make better compliance for patients, and reduce operation-associated complications. Despite the associated surgical complications being still high, correct understanding and reasonable treatment strategies can minimize the pain experienced by patients and improve the surgical success rate.

\section{Additional file}

Additional file 1: This is the raw data.(XLSX $14 \mathrm{~kb})$

\section{Abbreviations}

ASAMI: Association for the Study and Application of the Method of Ilizarov; DOG: Distraction osteogenesis; EFI: External fixation index; IM: Intra-medullary; JAMA: The Journal of the American Medical Association; LLD: Limb length discrepancy; TSF: Taylor spatial frame

\section{Acknowledgements}

This work is supported by the Chinese PLA general hospital, and we really appreciate the help given by related departments and participators.

Funding

No external funding was received.

\section{Availability of data and materials}

The datasets used and/or analyzed during the current study are available from the corresponding author on reasonable request.

\section{Authors' contributions}

ZQ was involved in overall study design and funding. ZQ and ZW contributed equally to this work and should be considered co-first authors. ZW, ZZ, ZLC, $\mathrm{DJH}$, and $\mathrm{HM}$ designed and wrote the analysis plan for the current paper. ZW undertook the statistical analyses. All authors were involved in the interpretation of data. ZQ and ZW wrote the first draft of the manuscript. All authors critically read the manuscript to improve intellectual content. All authors have approved the final manuscript in its present form.

Ethics approval and consent to participate

The study was approved by the Ethics Committee of Chinese PLA General Hospital, and written informed consent was obtained from the patients for the publication of individual clinical details and accompanying images. The study was conducted according to the ethical principles stated in the Declaration of Helsinki.

Consent for publication

All authors consent for publication.

Competing interests

The authors declare that they have no competing interests.

\section{Publisher's Note}

Springer Nature remains neutral with regard to jurisdictional claims in published maps and institutional affiliations.

Received: 18 April 2017 Accepted: 9 November 2017

Published online: 25 November 2017

References

1. Nikolaos KK, Theodoros HT, Peter VG. Surgical management of infected non-unions: an update. Injury. 2016;46:25-32. 
2. Kyu-Hyun Y, Yougun W, Sang BK, et al. Plate augmentation and autologous bone grafting after intramedullary nailing for challenging femoral bone defect: a technical note. Arch Orthop Trauma Surg. 2016;136:1381-5.

3. Anastasios DK, Panayaotis NS. Management of nonunion with distraction osteogenesis. Injury. 2006;37:51-5.

4. Ioannis DG, Angelos NP, Christina MA, et al. Diagnostic and treatment modalities in nonunions of the femoral shaft. a review. Injury. 2012;43:980-8.

5. Robert R S, Jacob SP, Austin TF, et al. Repair of tibial nonunions and bone defect with the Taylor spatial frame. J Orthop Trauma. 2008;22:88-95.

6. Wei FC, TA EI-G, Lin CH, Ueng WN. Free fibula osteoseptocutaneous graft for reconstruction of segmental femoral defects. J Trauma. 1997:43:784-92.

7. Yajima $\mathrm{H}$, Tamai $\mathrm{S}$, Mizumoto $\mathrm{S}$, One $\mathrm{H}$. Vascularised fibular grafts for reconstruction of the femur. J Bone Joint Surg (Br). 1993;75:123-8.

8. Masquelet AC, Begue T. The concept of induced membrane for reconstruction of long bone defects. Orthop Clin N Am. 2010:41:27-37.

9. Giannoudis, PV. Treatment of bone defects: bone transport or the induced membrane technique? Injury. 2016:47:291-2.

10. Papakostidis C, Bhandari M, Giannoudis PV. Distraction osteogenesis in the treatment of long bone defects of the lower limbs: effectiveness, complications and clinical results; a systematic review and meta analysis. Bone Joint J. 2013;95:1673-80.

11. Wael A, Mohamed ES. llizarov distraction osteogenesis over the preexisting nail for treatment of nonunited femurs with significant shortening. Eur J Orthop Surg Traumatol. 2016;26:319-28.

12. Saridis A, Panagiotopoulos E, Tyllianakis M, et al. The use of the llizarov method as a salvage procedure in infected nonunion of the distal femur with bone loss. J Bone Joint Surg (Br). 2006;88:232-7.

13. Kucukkaya M, Karakoyun O, Armagan R, Kuzqun U. Correction of complex lower extremities deformities with use of the llizarov-Taylor spatial frame. Acta Orthop Traumatol Turc. 2009;43:1-6.

14. Khanfour AA, Mohamed ME. Efficacy of a compliant semicircular Ilizarov pin fixator module for treating infected nonunion of the femoral diaphysis. Strat Traum Limb Recon. 2014:9:101-9.

15. Chanchit $\mathrm{S}$. Distraction osteogenesis for the treatment of post traumatic complications using a conventional external fixator: a novel technique. Injury. 2005;36:185-93.

16. Raj KH, Sohan SS, Divesh J. Management of nonunion of lower-extremity long bones using mono-lateral external fixator-report of 37 cases. Injury. 2014;45:560-7.

17. Sailhan F. Bone lengthening (distraction osteogenesis): a literature review. Osteoporos Int. 2011;22:2011-5.

18. Peng $Y$, Lihai $Z$, Tongtong $L$, et al. Infected nonunion of tibia and femur treated by bone transport. J Orthop Surg Res. 2015;10:49-59.

19. Marsh JL, Nepola JV, Mefffert R. Dynamic external fixation for stabilization of non union. Clin Orthop. 1992;278:200-6.

20. Paley D, Catagni MA, Argnani F. Ilizarov treatment of tibial nonunions with bone loss. Clin Orthop. 1989;241:146-65

21. Robert Z, Ze X, Thomas $E$, et al. Epidemiology of fracture nonunion in 18 human bones. JAMA Surgery. 2016;9:1-12.

22. Hou SM, Liu TK. Reconstruction of skeletal defects in the femur with "twostrut" free vascularized fibular grafts. J Trauma. 1992;33:840-5.

23. Olesen UK, Eckardt H, Bosemark P, et al. The Masquelet technique of induced membrane for healing of bone defects: a review of 8 cases. Injury. 2015;46:44-7.

24. Rohilla R, Wadhwani J, Devag A, et al. Prospective randomized comparison of ring versus rail fixator in infected gap nonunion of tibia treated with distraction osteogenesis. Bone Joint J. 2016;10:1399-405.

25. Blum AL, BongioVanni JC, Morgan SJ, et al. Complications associated with distraction osteogenesis for infected nonunion of the femoral shaft in the presence of a bone defect: a retrospective series. J Bone Joint Surg. 2010;92: $565-70$

26. Nikolaos G, Badri N, Selvadurai N. Distraction osteogenesis and nonunion of the docking site: is there an ideal treatment option? Injury. 2007;38:100-7.

27. Asim MM, Adrian SC, Juan SR, et al. The accordion maneuver: a noninvasive strategy for absent or delayed callus formation in cases of limb lengthening. Adv Orthop. 2015;3:1-8.

28. Paley D, Maar DC. llizarov bone transport treatment for tibial defects. J Orthop Trauma. 2000;14:76-85

\section{Submit your next manuscript to BioMed Central and we will help you at every step:}

- We accept pre-submission inquiries

- Our selector tool helps you to find the most relevant journal

- We provide round the clock customer support

- Convenient online submission

- Thorough peer review

- Inclusion in PubMed and all major indexing services

- Maximum visibility for your research

Submit your manuscript at www.biomedcentral.com/submit
Biomed Central 